\title{
Reply to Di Donato and colleagues
}

\author{
James 0. O'Neill, FRCPI, ${ }^{\mathrm{c}}$ Randall C. Starling, FACC, MPH, ${ }^{\mathrm{b}}$ and Mina K. Chung, FACC $^{\mathrm{a}}$
}

See related editorial on page 289.

From the Departments of Cardiac Electrophysiology, ${ }^{a}$ Cleveland Clinic Foundation, Cleveland, Ohio, and Heart Failure and Cardiac Transplantation, ${ }^{\mathrm{b}}$ Cleveland Clinic Foundation, Cleveland, Ohio, and the Department of Clinical Cardiology, ${ }^{\mathrm{c}}$ Conolly Hospital, Dublin, Ireland.

Mina Chung reports consulting and lecture fees from Medtronic and Guidant and grant support from Medtronic. Randall Starling reports consulting fees from Medtronic.

Received for publication Sept 9, 2006; accepted for publication Sept 20, 2006.

Address for reprints: Randall C. Starling, FACC, MPH, Department of Heart Failure and Cardiac Transplantation, Cleveland Clinic Foundation, Cleveland, Ohio, 44118 (E-mail: starlir@ccf.org).

J Thorac Cardiovasc Surg 2007;133:292-3

$0022-5223 / \$ 32.00$

Copyright $\odot 2007$ by The American Association for Thoracic Surgery

doi:10.1016/j.jtcvs.2006.09.063
$\mathrm{W}$

e are grateful to the editors of the Journal for allowing us to respond to the editorial by Di Donato and colleagues. ${ }^{1}$

We wish to acknowledge the pioneering work by Drs Di Donato, Sabatier, and Mericanti and in particular Dr Dor in the field of surgery for patients with heart failure.

Their editorial raises several important issues. At the heart of the debate is whether left ventricular reconstruction is proarrhythmogenic, antiarrhythmogenic, or completely neutral. Dr Di Donato and colleagues have the largest collection of patients who have undergone volume reduction surgery, and they have produced many sentinel articles in this field.

The differences in outcomes can be explained by several factors. In our cohort patients were at a more advanced stage of disease, with mean left ventricular ejection fractions of $22 \%$ compared with a median of $29 \%$ in Di Donato's group. ${ }^{2}$ We agree that major differences in arrhythmia occurrence could reflect the use of endocardial resection with cryoablation in the Dor series, and as noted, cryoablation was performed in a minority of our patients. However, the patients referred to in our study underwent surgical intervention as part of a comprehensive approach to the management of their heart failure syndrome. They were not referred primarily for aneurysmectomy on the basis of arrhythmia, an indication that led to left ventricular reconstructive surgery in a significant number of this group's series. ${ }^{3}$ The arrhythmia management was based on electrophysiologic testing or implantable cardioverter defibrillator implantation, with the latter approach well supported by several recent studies showing the efficacy of implantable cardioverter defibrillators in the primary prevention of sudden death in patients with ischemic heart disease.

We do not perform electrophysiological studies routinely preoperatively because we support the hypothesis that revascularization and improved left ventricular geometry with reduced wall stress should act to reduce the substrate for arrhythmia. Thus apart from a research perspective, there appears to be no potential benefit from preoperative studies. The differences in postoperative inducibility might well be related to our lower use of endocardial resection and cryoablation. We also agree that the use of a maximum of 2 extrastimuli during programmed stimulation would be less sensitive in inducing ventricular arrhythmias than our usual stimulation protocol of up to 3 extrastimuli at 2 right ventricular sites. We have also used a protocol with 4 extrastimuli, which has been reported to yield equivalent inducibility rates to those of conventional protocols with 3 extrastimuli. ${ }^{4}$

We do not use amiodarone routinely as performed in the authors' practice. The use of amiodarone for the primary prevention of arrhythmias is of questionable benefit, particularly in light of the SCD HeFT II study, which showed increased mortality with amiodarone compared with placebo, albeit in a different patient population from that described here. ${ }^{5}$

We acknowledge that left ventricular volumes would be very telling in assessing outcome, but we did not have sufficient magnetic resonance imaging data to address this, and patients with devices did not undergo magnetic resonance imaging.

In summary, our data indicate that patients with severe heart failure who undergo left ventricular reconstruction remain at high risk of malignant ventricular arrhythmias. We cannot nor did we conclude that the risk was higher than preoperatively. 
We believe that the difference in ventricular arrhythmias can be explained by the patient profile, specifically that our patients had more advanced disease with poorer left ventricular ejection fractions. We would make a case for the judicious use of adjunctive device therapy in patients undergoing left ventricular reconstruction. Only a small percentage of our patients had an improvement in left ventricular ejection fraction above the MADIT II cutoff of 30\%. ${ }^{6}$

\section{References}

1. Di Donato M, Sabatier M, Menicanti L, Dor V. Incidence of ventricular arrhythmias after left ventricular reconstructive surgery. $J$ Thorac Cardiovasc Surg. 2006;133:289-91.
2. O'Neill JO, Starling RC, Khaykin Y, et al. Residual high incidence of ventricular arrhythmias after left ventricular reconstructive surgery. J Thorac Cardiovasc Surg. 2005;130:1250-6.

3. Di Donato M, Sabatier M, Dor V. Surgical ventricular restoration in patients with postinfarction coronary artery disease: effectiveness on spontaneous and inducible ventricular tachycardia. Semin Thorac Cardiovasc Surg. 2001;13:480-5.

4. Hummel JD, Strickberger SA, Daoud E, et al. Results and efficiency of programmed ventricular stimulation with four extrastimuli compared with one, two, and three extrastimuli. Circulation. 1994;90:2827-32.

5. Bardy GH, Lee KL, Mark DB, et al. Amiodarone or an implantable cardioverter-defibrillator for congestive heart failure. $N$ Engl $\mathrm{J} \mathrm{Med}$. 2005;352:225-37.

6. Moss AJ, Zareba W, Hall WJ, et al. Prophylactic implantation of a defibrillator in patients with myocardial infarction and reduced ejection fraction. N Engl J Med. 2002;346:877-83. 